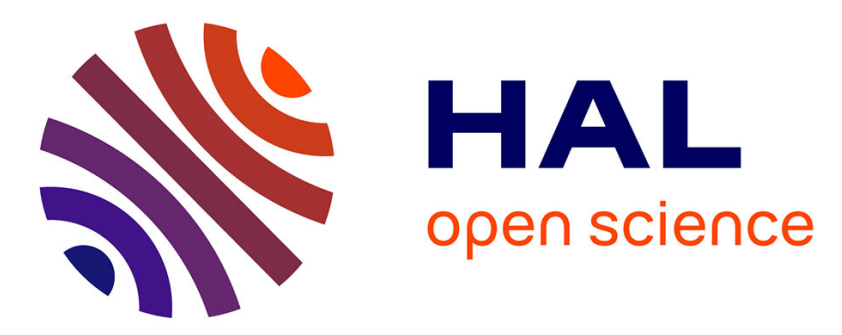

\title{
On the reduction and rectification of thermal conduction using phononic crystals with pacman-shaped holes
}

Sergei Gluchko, Roman Anufriev, Ryoto Yanagisawa, Sebastian Volz, Masahiro Nomura

\section{- To cite this version:}

Sergei Gluchko, Roman Anufriev, Ryoto Yanagisawa, Sebastian Volz, Masahiro Nomura. On the reduction and rectification of thermal conduction using phononic crystals with pacman-shaped holes. Applied Physics Letters, 2019, 114, 10.1063/1.5079931 . hal-02364610

\section{HAL Id: hal-02364610 https://hal.science/hal-02364610}

Submitted on 15 Nov 2019

HAL is a multi-disciplinary open access archive for the deposit and dissemination of scientific research documents, whether they are published or not. The documents may come from teaching and research institutions in France or abroad, or from public or private research centers.
L'archive ouverte pluridisciplinaire HAL, est destinée au dépôt et à la diffusion de documents scientifiques de niveau recherche, publiés ou non, émanant des établissements d'enseignement et de recherche français ou étrangers, des laboratoires publics ou privés. 


\section{On the reduction and rectification of thermal conduction using phononic crystals with pacman-shaped holes}

Cite as: Appl. Phys. Lett. 114, 023102 (2019); https://doi.org/10.1063/1.5079931

Submitted: 05 November 2018 . Accepted: 30 December 2018 . Published Online: 15 January 2019

Sergei Cluchko (D), Roman Anufriev (D), Ryoto Yanagisawa (D), Sebastian Volz (D), and Masahiro Nomura (D)
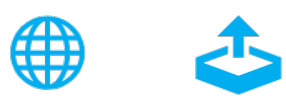


\title{
On the reduction and rectification of thermal conduction using phononic crystals with pacman-shaped holes
}

\author{
Cite as: Appl. Phys. Lett. 114, 023102 (2019); doi: 10.1063/1.5079931 \\ Submitted: 05 November 2018 - Accepted: 30 December 2018 - Published Online: \\ 15 January 2019 \\ Sergei Gluchko, ${ }^{1,2}$ (D) Roman Anufriev, ${ }^{1}$ (D) Ryoto Yanagisawa, (iD Sebastian Volz, ${ }^{1,2}$ (D) and Masahiro Nomura,3,a) (D) \\ AFFILIATIONS \\ ${ }^{1}$ Institute of Industrial Science, The University of Tokyo, Tokyo 153-8505, Japan \\ ${ }^{2}$ Laboratory for Integrated Micro Mechatronic Systems/National Center for Scientific Research-Institute of Industrial Science \\ (LIMMS/CNRS-IIS), The University of Tokyo, Tokyo 153-8505, Japan \\ ${ }^{3}$ PRESTO, Japan Science and Technology Agency, Kawaguchi, Saitama 332-0012, Japan \\ a) Author to whom correspondence should be addressed: nomura@iis.u-tokyo.ac.jp
}

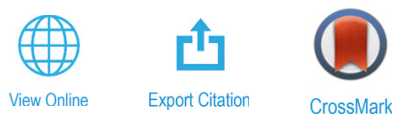

\begin{abstract}
We measure the thermal conductivity of silicon phononic crystals with asymmetric holes at room and liquid helium temperatures and study the effect of thermal rectification, phonon boundary scattering, neck transmission, and hole positioning. Also, we compare the influence of asymmetric holes on thermal conductivity reduction with the one of conventional circular holes. This reduction is almost $40 \%$ larger in the case of pacman shaped holes as compared with circular ones for the same parameters of phononic crystals. Our experimental results can be used to significantly improve the efficiency of thermoelectric devices by using pacman-shaped holes in phononic crystals.
\end{abstract}

Published under license by AIP Publishing. https://doi.org/10.1063/1.5079931

Development of modern wireless microelectronics requires autonomous power generators and thermoelectric devices offer a convenient solution when sunlight and mechanical vibrations are not available. However, the integration with CMOS technology suggests us to use thermoelectric generators based on silicon, which is a poor thermoelectric material. ${ }^{1,2}$ In the past decade, researchers demonstrated that the thermoelectric efficiency of silicon can be increased by nanostructuring., Particularly, one method to enhance the thermoelectric efficiency is to reduce the thermal conductivity of the structure by using thin membranes with arrays of holes, called phononic crystals (PnCs). ${ }^{5-7}$ In such PnCs, the holes scatter phonons-the main heat carriers in silicon-and thus suppress heat conduction. Moreover, theoretical works propose that structures with asymmetric holes $^{8}$ or boundaries ${ }^{9}$ can create a thermal rectification effect, which would further benefit thermoelectric devices.

A recent experimental study ${ }^{10}$ could not verify the geometry induced thermal rectification effect obtained by Schmotz et al. ${ }^{11}$ but suggested that the non-circular holes might be more efficient in the thermal conductivity reduction than conventional circular holes. Also, experiments show that the surface area of holes may play a key role in the reduction of thermal conductivity ${ }^{12,13}$ but only above a certain nanoscale limit, below which narrow passages between the holes take over the control of thermal conductivity. ${ }^{6,12,14,15}$ However, the exact roles of hole symmetry, surface area, size, and separation in the thermal conductivity reduction remain unclear.

In this letter, we systematically study the reduction of thermal conductivity in PnCs with asymmetric holes of various shapes and dimensions. We show that asymmetric holes produce no thermal rectification but reduce the thermal conductivity more efficiently than circular holes. We distinguish two mechanisms of this reduction: diffusive surface scattering of phonons and phonon scattering at the narrow passages between the holes. Understanding of these mechanisms allows us to design the PnCs that reduce thermal conductivity as much as conventional PnCs with circular holes but without sacrificing the same amount of material volume.

All our samples were fabricated on the same 70-nm-thick (100) undoped silicon-on-insulator (SOI) wafer using the topdown technique. Figure 1 shows scanning electron microscopy 
images of a typical sample with arrays of holes. Metal pads and heat sinks of $5 \times 5 \mu \mathrm{m}^{2}$ and $25 \times 25 \mu \mathrm{m}^{2}$ were fabricated by using electron beam lithography and electron beam-assisted metal evaporation of a $70 \mathrm{~nm}$ thick aluminum layer, respectively. The arrays of holes were fabricated by electron beam lithography and cryogenic inductively coupled plasma reactive-ion etching (ICP RIE) processes providing low boundary roughness as well as high aspect ratio of the holes. The suspension of the membranes with PnCs was achieved by removing the $2 \mu \mathrm{m}$ buried oxide layer of SOI wafer via hydrofluoric acid vapor. The suspension length and width of all the bridges were set to $30 \mu \mathrm{m}$.

Thermal conductivity measurements were performed by means of the micro time-domain thermoreflectance technique ( $\mu$ TDTR). This method is based on measuring the aluminum pad reflectance variation by a probe laser while heating it with a pump laser operating in a pulse regime. The temperature increase in the aluminum pad changes the amount of light that is reflected from its surface as a result of temperature dependence of the aluminum dielectric constant. The aluminum pad itself is fabricated in the middle of a suspended silicon membrane bridge structure with a PnC supported by two heat sinks. The heat that is generated by the pulse of a pump laser in the central metal pad propagates along the suspended PnCs and dissipates into the heat sinks. By analyzing the decay time of the structures, we extract the thermal conductivity using finite element heat transfer simulations. We use the approach of porous media where the porosity of each PnC is measured using SEM and its thermal conductivity is corrected by its porosity using the Eucken mode ${ }^{16}$ as follows:

$$
\kappa=\kappa_{\text {porous }} \frac{1+0.5 \phi}{1-\phi},
$$

where $\kappa_{\text {porous }}$ is the thermal conductivity of a porous membrane used for finite element simulations of a PnC and $\phi$ is an experimentally measured porosity. This approach allows us to take

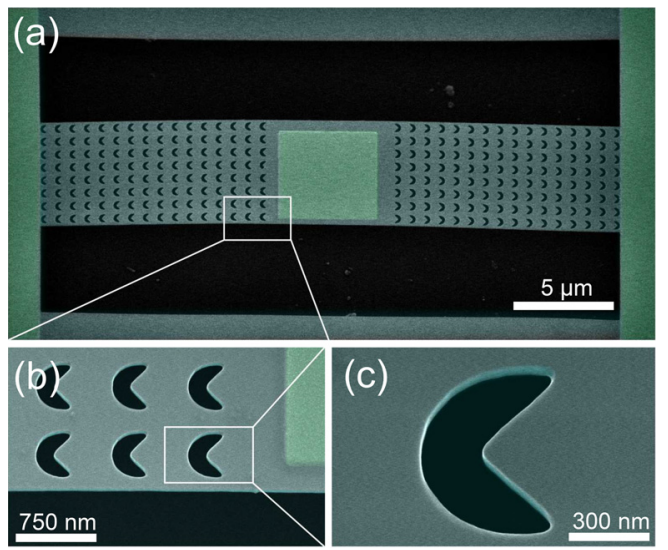

FIG. 1. Tilted SEM image of a phononic crystal with pacman type holes. The vertical neck size is $250 \mathrm{~nm}$, the hole radius is $250 \mathrm{~nm}$, the period is $750 \mathrm{~nm}$, and the silicon membrane thickness is $70 \mathrm{~nm}$. (a) Top view of a suspended silicon membrane with an aluminum pad and two heat sinks (green color), magnified images of an array with holes (b), and individual pacman hole (c). into account fabrication imperfections due to a limited resolution of electron beam lithography. It is necessary when comparing thermal conductivity of $\mathrm{PnC}$ with complex hole shapes. Figure 2 shows a typical TDTR signal obtained by averaging $10^{5}$ rectangular pulses of a pump laser measured at ambient temperature. The inset of Fig. 2 shows a simplified schematic of the setup. All our measurements were conducted under vacuum to prevent the influence of air convection. We used three independent samples with exactly the same parameters for each measurement in order to reduce the influence of mechanical vibrations of the membranes and the errors induced by fabrication uncertainties. ${ }^{1}$

First, we examine the influence of the orientation of asymmetric holes on heat conduction at 4.2 and $295 \mathrm{~K}$. Figures 3(a) and $3(\mathrm{~b})$ show the forward and backward orientations of the pacman holes with respect to the metal pad used for $\mu$ TDTR measurements. In the case of backward orientation, one should expect lower thermal conductivity due to a possibility of two specular boundary scattering events. Indeed, if a phonon experiences two specular reflections from the boundaries of the pacman's "mouth" during its free pass, it should be reflected backwards independently of its initial direction. In the case of the forward direction, the phonon should be specularly reflected in a random direction depending on its incident direction. As a result, such a PnC should behave as a thermal diode. Figure 3(c) shows experimentally measured thermal conductivity in both orientations of the holes at room temperature. We observe no thermal rectification effect within the sensitivity of our experimental setup for such a system at $\mathrm{T}=295 \mathrm{~K}$.

The mean free path of phonons in Si ranges from few tens of nanometers to several microns ${ }^{12,18,19}$ and the effect of thermal conductivity rectification should be observed only for small size

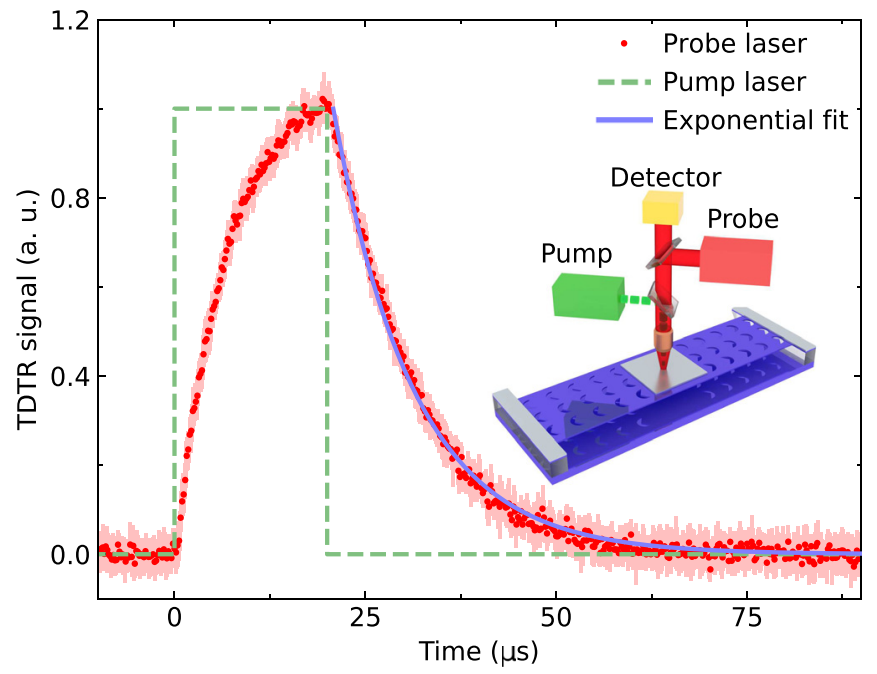

FIG. 2. $\mu$ TDTR signal obtained for a $\mathrm{PnC}$ with pacman $100 \mathrm{~nm}$ holes at $T=295 \mathrm{~K}$. The green dashed line indicates the supply voltage of a pump laser modulated with $20 \mu \mathrm{s}$ rectangular pulses. Red dots show the reflected signal of a probe laser, magenta bars indicate the standard deviation of the probe signal, and the blue line is an exponential fitting of the probe signal decay. The inset shows the simplified schematic of the thermoreflectance measurement setup and a sample. 

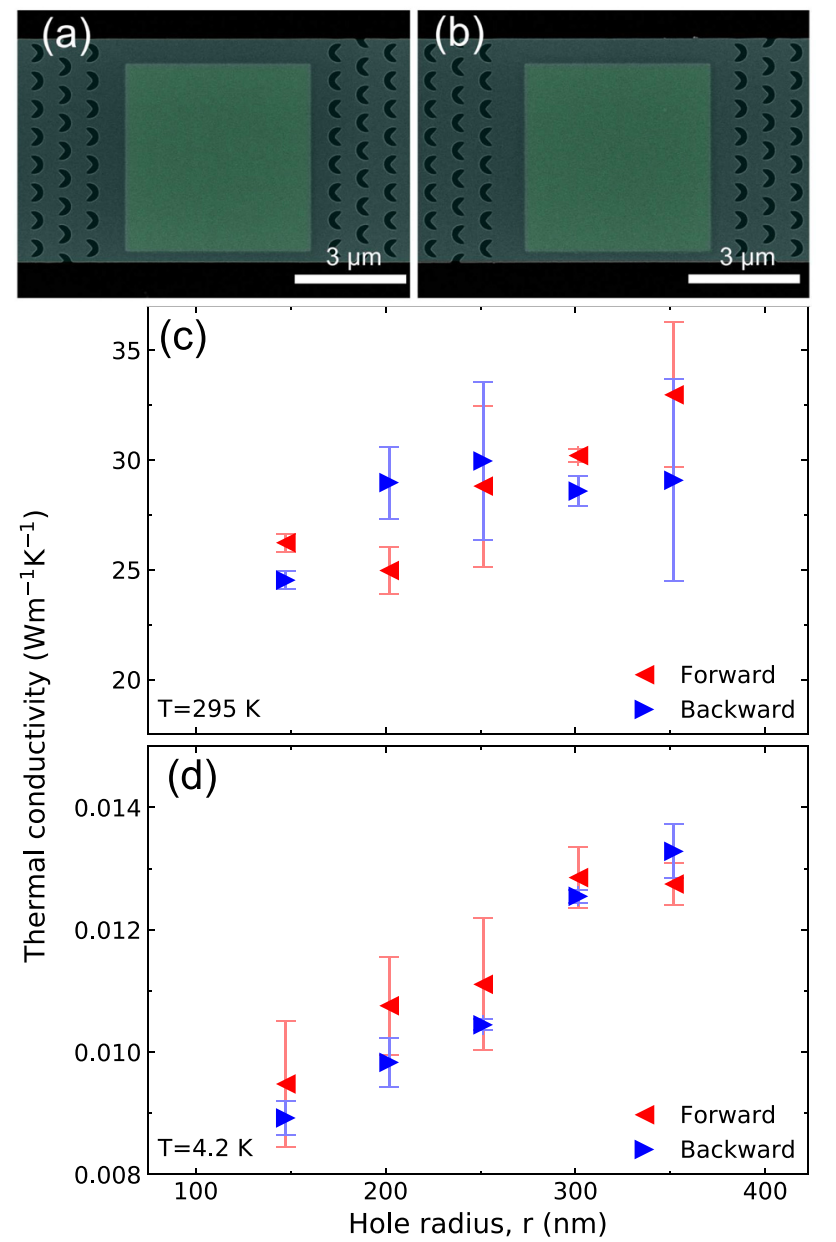

FIG. 3. SEM image of the central part of the bridge with the aluminum pad and pacman PnC for forward (a) and backward (b) orientations of the holes. In-plane thermal conductivity of pacman $\mathrm{PnC}$ measured at room temperature (c) and at $4.2 \mathrm{~K}$ (d). The neck size is equal to the hole radius for all the PnCs.

holes when the size of the hole is significantly smaller than phonon mean free paths contributing the most to material thermal conductivity. However, fabrication of smaller holes with a complex shape remains difficult due to a limited resolution of electron beam lithography. Another way to increase the probability of specular boundary scattering of the phonon is to decrease the temperature of the system because phonon mean free paths and wavelengths are longer at low temperatures. Figure 3(d) shows thermal conductivity of PnCs with pacman type holes at $\mathrm{T}=4.2 \mathrm{~K}$. Backward oriented holes systematically show slightly lower thermal conductivity for a small size of the holes. Although this could be a signature of the thermal diode effect, this difference is smaller than the standard deviation of the measurement and could not be detected reliably with our current setup.

The thermal conductivity reduction due to pacman PnCs, shown in Fig. 3(c), is stronger than the reduction usually obtained for similar PnCs with circular holes. ${ }^{12}$ We attribute this stronger thermal conductivity reduction to a larger scattering surface of pacman holes as compared with circular ones for the same hole radius and neck size.

To study the impact of the hole shape in more detail, we measure the thermal conductivity in PnCs with holes of three different shapes: pacman [Fig. 4(b)], long pacman [Fig. 4(c)], and circular [Fig. 4(d)]. For all our samples, we keep the PnC periodto-radius ratio the same $a / r=3$. The distance between two neighbor holes is equal to the radius of the hole, as indicated in Fig. 4(a). The circular shaped hole has the smallest possible surface-to-volume ratio $\frac{S}{V}=2 / r$ and the same fixed porosity for different hole radii $\phi_{\text {cir }}=\pi / 9 \approx 0.35$. The pacman shaped hole has the largest surface-to-volume ratio $\frac{\mathrm{S}}{\mathrm{V}} \approx 3.44 / r$ with half of the porosity of the circular $\mathrm{PnC} \phi_{\mathrm{pac}}=0.5 \phi_{\mathrm{cir}} \approx 0.175$. In the case of a long pacman hole, the porosity is equal to the one of $\mathrm{PnC}$ with circular holes $\phi_{\text {lpac }}=0.5 \phi_{\text {cir }} \approx 0.35$, while the surface-to-volume
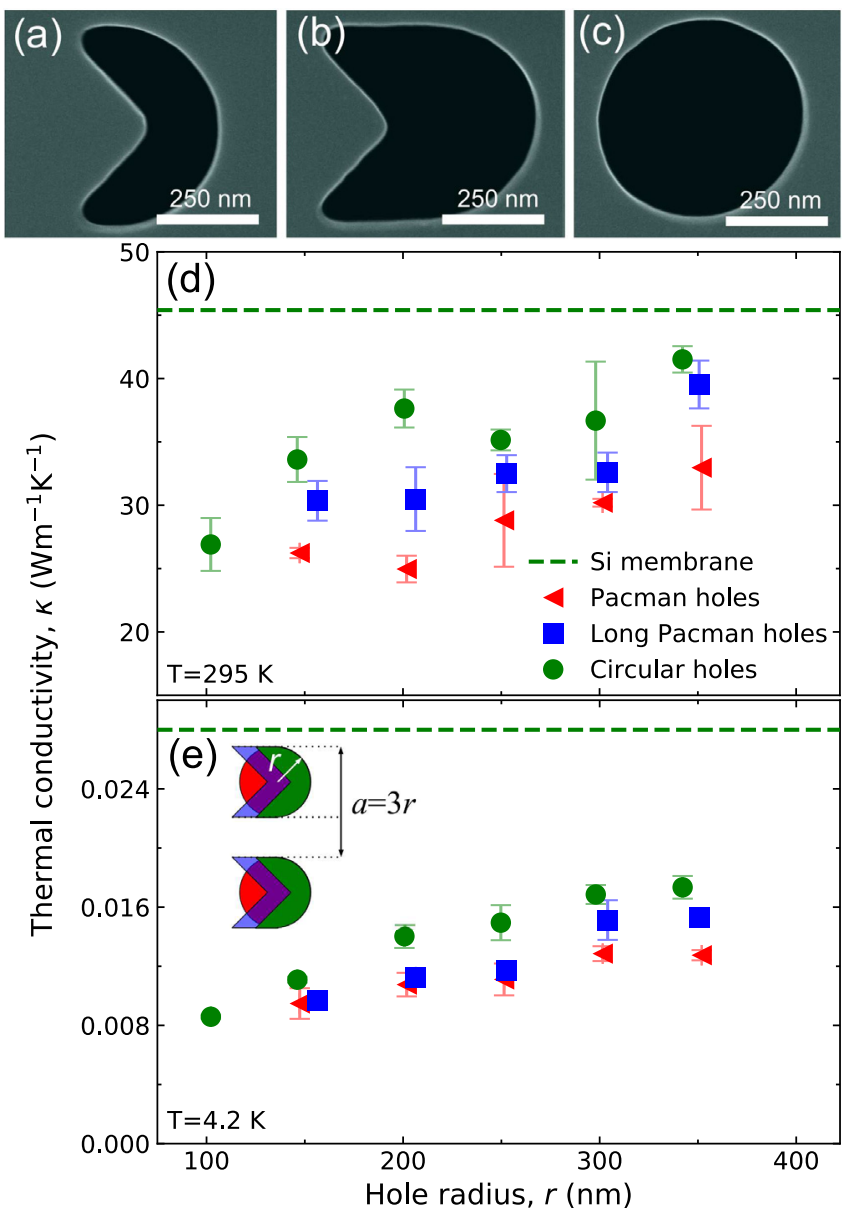

FIG. 4. SEM image of a pacman type hole (a), long pacman type hole (b), and circular type hole (c). In-plane thermal conductivity of $\mathrm{PnC}$ with different types of holes as a function of their radius measured at room temperature (d) and at $4.2 \mathrm{~K}(\mathrm{e})$. The neck size is equal to the hole radius for all the PnCs. The green dashed line indicates thermal conductivity of 70-nm-thick membranes without holes. The inset in (e) shows the relative dimensions of all types of holes and their hole radius. For all samples, the period to hole radius ratio is fixed $a / r=3$. 
ratio $\frac{S}{V} \approx 2.71 / r$ is smaller than the one of the pacman hole. Figure 4(d) shows experimentally measured thermal conductivity of all three types of PnCs at room temperature. The neck is equal to the hole radius for all the PnCs. Pacman holes demonstrate the largest thermal conductivity reduction among these three shapes for all hole sizes. Due to its highest surface-to-volume ratio, pacman hole PnC is almost $40 \%$ less thermally conductive than circular hole PnC. However, at low temperatures, at which the phonon wavelengths are longer than the surface roughness, the difference in thermal conductivity almost disappears for small neck sizes. This behaviour indicates that the boundary scattering of phonons is predominant at room temperatures, while the neck effect is predominant at low temperatures. This separation of the neck effect and boundary scattering can be used independently to control the thermal conductivity of PnC.

Independent ways to control material thermal conductivity can be used simultaneously to design high efficiency materials.
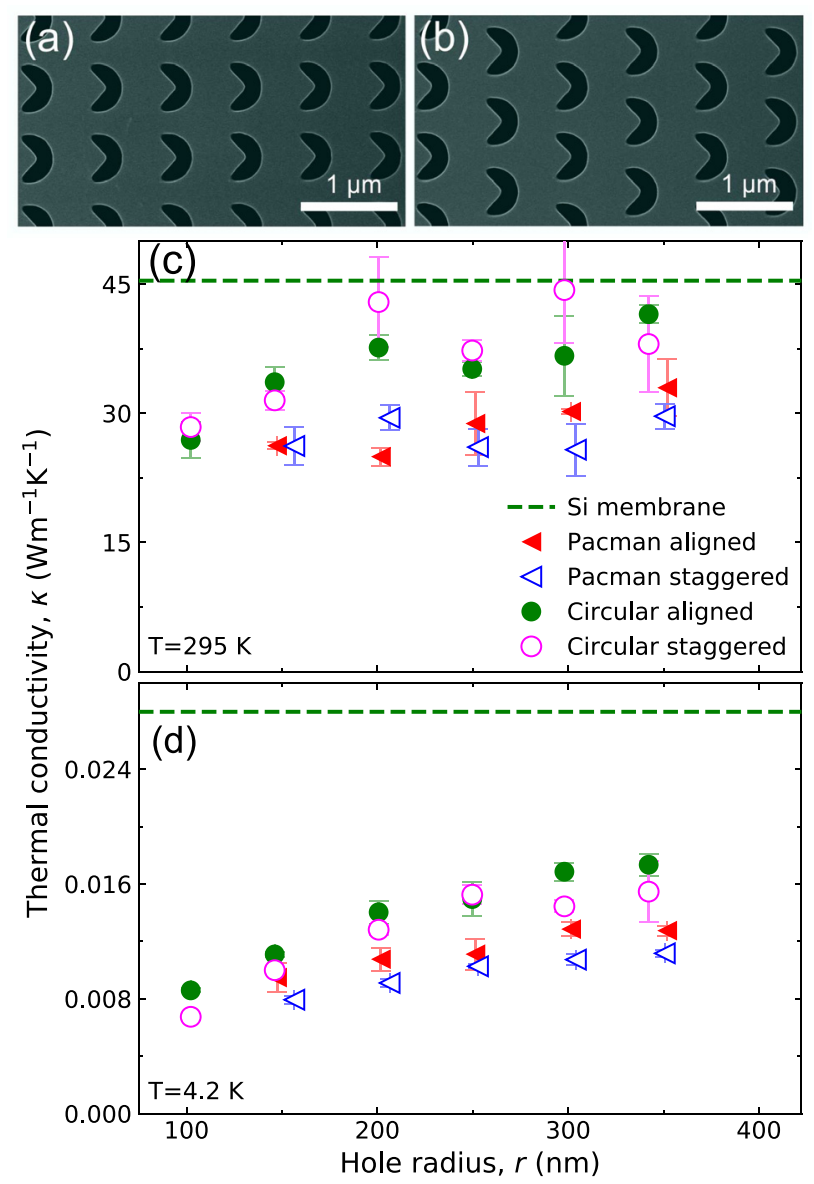

FIG. 5. SEM images of a pacman type PnC with aligned (a) and staggered (b) lattice types. In-plane thermal conductivity of PnCs with circular and pacman types of holes of aligned and staggered lattices as a function of their radius, measured at room temperature (c) and at $4.2 \mathrm{~K}(\mathrm{~d})$. The neck size is equal to the hole radius for all the PnC. The green dashed line indicates the thermal conductivity of 70 -nm-thick membranes without holes.
Figures 5(a) and 5(b) show SEM images of aligned (square) and staggered lattice types of pacman PnCs. The staggered lattice is obtained from an aligned lattice with period $a=3 r$ by translating every second column by half of the lattice period along the vertical axis. The staggered type of PnC lattice can prevent directional phonon contribution and significantly lowers PnC thermal conductivity as compared to the aligned lattice type. ${ }^{20,21}$ Combining the effects of thermal conductivity reduction due to the phonon boundary scattering on complex shapes, neck effect, and phonon directionality opens a possibility to create a material with even lower thermal conductivity. Figures 5(c) and 5(d) show experimentally measured thermal conductivity of aligned and staggered PnCs with pacman and circular shaped holes for room and $4.2 \mathrm{~K}$ temperatures, respectively. Pacman shape staggered PnC demonstrates almost twice lower thermal conductivity than a silicon membrane without holes.

In this work, we have observed through experiment reduction of in-plane thermal conductivity of silicon phononic crystals with three different types of holes. We found no thermal rectification effect for strongly asymmetric holes at room temperature. At $4.2 \mathrm{~K}$, we could observe a signature of the thermal diode effect but not exceeding the instrumental uncertainty. Development of thermal rectification in a monolithic material with nanostructuring can be useful for many applications and requires further investigations. We have also demonstrated that boundary scattering of phonons can be controlled separately from the neck effect by changing the shape of the holes of PnCs and their surface-to-volume ratio. PnCs with pacman holes in the staggered lattice provide almost $40 \%$ lower thermal conductivity than the one with circular holes of the same radius and period and almost $50 \%$ lower thermal conductivity than of a silicon membrane without holes. At room temperature, PnCs with a large surface-to-volume ratio reduce thermal conductivity more efficiently even for the same PnC period and neck size. At low temperatures, the dominant mechanism of $\mathrm{PnC}$ thermal conductivity reduction is the neck effect. We believe that the separation of these two mechanisms of PnC thermal conductivity reduction should open possibilities for improvement of thermoelectric materials design as well as provide tools for heat manipulation at the nanoscale.

This work was supported by Kakenhi (Nos. 15H05869, 15K13270, and 18K14078), PRESTO JST (No. JPMJPR15R4), and the Postdoctoral Fellowship Program of Japan Society for the Promotion of Science (No. 18F18725).

\section{REFERENCES}

${ }^{7}$ M. Haras, V. Lacatena, T. M. Bah, S. DIdenko, J. F. Robillard, S. Monfray, T. Skotnicki, and E. Dubois, IEEE Electron Device Lett. 37, 1358 (2016).

${ }^{2}$ G. Schierning, Phys. Status Solidi A 211, 1235 (2014).

${ }^{3}$ A. Minnich, M. S. Dresselhaus, Z. F. Ren, and G. Chen, Energy Environ. Sci. 2, 466 (2009).

${ }^{4}$ K. Nielsch, J. Bachmann, J. Kimling, and H. Böttner, Adv. Energy Mater. 1, 713 (2011).

${ }^{5}$ M. Nomura, J. Shiomi, T. Shiga, and R. Anufriev, Jpn. J. Appl. Phys. 57, $080101(2018)$

${ }^{6}$ J. Lim, H.-T. T. Wang, J. Tang, S. C. Andrews, H. So, J. Lee, D. H. Lee, T. P. Russell, and P. Yang, ACS Nano 10, 124 (2016). 
${ }^{7}$ J. Tang, H.-T. Wang, D. H. Lee, M. Fardy, Z. Huo, T. P. Russell, and P. Yang, Nano Lett. 10, 4279 (2010).

${ }^{8}$ Z. Yu, L. Ferrer-argemi, and J. Lee, J. Appl. Phys. 122, 244305 (2017).

${ }^{9} \mathrm{~N}$. Roberts and D. Walker, Int. J. Therm. Sci. 50, 648 (2011).

${ }^{10}$ Y. Kage, H. Hagino, R. Yanagisawa, J. Maire, K. Miyazaki, and M. Nomura, Jpn. J. Appl. Phys. 55, 085201 (2016).

"M. Schmotz, J. Maier, E. Scheer, and P. Leiderer, New J. Phys. 13, 113027 (2011).

${ }^{12}$ R. Anufriev, J. Maire, and M. Nomura, Phys. Rev. B 93, 045411 (2016).

${ }^{13}$ B. Graczykowski, A. El Sachat, J. S. Reparaz, M. Sledzinska, M. R. Wagner, E. Chavez-Angel, S. Volz, Y. Wu, F. Alzina, C. M. Sotomayor Torres, S. Volz, Y. Wu, F. Alzina, and C. M. Sotomayor Torres, Nat. Commun. 8, 415 (2017).
${ }^{14}$ R. Yanagisawa, J. Maire, A. Ramiere, R. Anufriev, and M. Nomura, Appl. Phys. Lett. 110, 133108 (2017).

${ }^{15}$ K. D. Parrish, J. R. Abel, A. Jain, J. A. Malen, and A. J. H. McGaughey, J. Appl. Phys. 122, 125101 (2017).

${ }^{16}$ A. Eucken, Forsch. Geb. Ingenieurwes. 11, 6 (1940).

${ }^{17}$ R. Anufriev, S. Gluchko, S. Volz, and M. Nomura, ACS Nano 12, 11928 (2018).

${ }^{18} \mathrm{M}$. Nomura, Microsyst. Technol. 22, 473 (2016).

${ }^{19}$ W. Park, J. Sohn, G. Romano, T. Kodama, A. Sood, J. S. Katz, B. S. Y. Kim, H. So, E. C. Ahn, M. Asheghi, A. M. Kolpak, and K. E. Goodson, Nanoscale 10, 11117 (2018).

${ }^{20}$ R. Anufriev, A. Ramiere, J. Maire, and M. Nomura, Nat. Commun. 8, 15505 (2017).

${ }^{21} \mathrm{M}$. Verdier, R. Anufriev, A. Ramiere, K. Termentzidis, and D. Lacroix, Phys. Rev. B 95, 205438 (2017). 